\title{
Range corrections in Proton Halo Nuclei
}

\author{
Emil Ryberg ${ }^{\mathrm{a}}$, Christian Forssén ${ }^{\mathrm{a}, \mathrm{d}, \mathrm{e}}$, H.-W. Hammer ${ }^{\mathrm{b}, \mathrm{c}}$, Lucas Platter ${ }^{\mathrm{a}, \mathrm{d}, \mathrm{e}, \mathrm{f}, *}$ \\ ${ }^{a}$ Department of Fundamental Physics, Chalmers University of Technology, \\ SE-412 96 Göteborg, Sweden \\ ${ }^{b}$ Institut für Kernphysik, Technische Universität Darmstadt, 64289 Darmstadt, Germany \\ ${ }^{c}$ ExtreMe Matter Institute EMMI, GSI Helmholtzzentrum für Schwerionenforschung, \\ 64291 Darmstadt, Germany \\ ${ }^{d}$ Department of Physics and Astronomy, University of Tennessee, Knoxville, TN 37996, \\ USA \\ ${ }^{e}$ Physics Division, Oak Ridge National Laboratory, Oak Ridge, TN , USA \\ ${ }^{f}$ Physics Division, Argonne National Laboratory, Argonne, Illinois 60439, USA
}

\begin{abstract}
We analyze the effects of finite-range corrections in halo effective field theory for S-wave proton halo nuclei. We calculate the charge radius to next-toleading order and the astrophysical S-factor for low-energy proton capture to fifth order in the low-energy expansion. As an application, we confront our results with experimental data for the S-factor for proton capture on Oxygen-16 into the excited $1 / 2^{+}$state of Fluorine-17. Our low-energy theory is characterized by a systematic low-energy expansion, which can be used to quantify an energy-dependent model error to be utilized in data fitting. Finally, we show that the existence of proton halos is suppressed by the need for two fine tunings in the underlying theory.
\end{abstract}

Keywords: halo nuclei, charge radius, radiative capture, effective field theory

\section{Introduction}

The quantitative description of both nuclear structure and reactions on the same footing is a major challenge of contemporary nuclear theory. With new experimental facilities such as FRIB and FAIR at the horizon, the task to find improved approaches for nuclear reactions has become very urgent. $A b$

*lplatter@utk.edu

Preprint submitted to Annals of Physics

January 6, 2016

(C) 2016. This manuscript version is made available under the Elsevier user license http://www.elsevier.com/open-access/userlicense/1.0/ 
initio approaches to calculate nuclear scattering observables are limited by the computational complexity of the nuclear many-body problem. Scattering models perform well but use a number of uncontrolled approximations that make the errors of such calculations difficult to quantify.

Faced with these problems, it is important to note that there are a number of systems in the chart of nuclei for which the effective number of degreesof-freedom is significantly smaller than the number of nucleons. This phenomenon is known as clustering, with alpha clustering, e.g. in the Hoyle state of ${ }^{12} \mathrm{C}$, being the most prominent example. Clustering becomes even more extreme for so-called halo nuclei which consist of a tightly-bound core nucleus and a few weakly-bound valence nucleons $[1,2,3,4]$. This reduction in the number of degrees of freedom is the signature of a separation of scales in the system. In the case of a one-nucleon halo nucleus, the scale separation is manifest in the small ratio of the one-nucleon separation energy and the binding and excitation energies of the core. For typical momenta on the order of the one-nucleon separation energy, it allows for a systematic low-energy expansion in the ratio of these two scales. This expansion can then be employed to calculate nuclear observables in a model-independent and systematically improvable manner. This approach is called halo effective field theory (Halo EFT) [5] when a field-theoretical approach is used for the construction of the interaction and the calculation of observables. Halo EFT employs the minimal number of degrees-of-freedom (core and valence nucleons) and parameterizes the interaction in terms of a few measurable parameters. In addition, so-called core polarization effects become important if the core has low-lying excited states. These can be taken into account by including excited states of the core as explicit degrees of freedom in the effective theory.

Neutron halo nuclei occur rather frequently in the chart of nuclei along the neutron dripline and the structure and reactions of a number of known and predicted one- and two-neutron halo systems in the Helium $[5,6,7,8]$, Lithium [9, 10, 11, 12, 13], Beryllium [9, 14, 13], Carbon [9, 15, 16, 17, 13], and Calcium isotope chains [18] have been studied in Halo EFT.

Proton halo systems exist too, but are less common due to the delicate interplay between attraction from the strong interaction and the repulsion from the Coulomb interaction. The effects of the Coulomb interaction were first included into an EFT with contact interactions by Kong and Ravndal [19]. Proton halos were considered recently in Refs. [20, 21, 22]. The Coulomb interaction introduces a new scale into the problem that can be understood 
as a result of the presence of a Coulomb barrier. This new scale is refered to as the Coulomb momentum and it is given by the inverse of the Bohr radius of the system. The introduction of a Coulomb momentum can complicate the power counting since it interferes with the separation of scales. Higa et al. [23], e.g., treated the Coulomb momentum as a high-momentum scale in their study of $\alpha-\alpha$ scattering. However, this treatment is not always appropriate. The correct scaling of the Coulomb momentum will always depend on the system to be considered.

In this paper, we will extend the calculation performed in Ref. [20] by including higher-order effects due to the finite range of the interaction between core and proton. We also consider higher-order electromagnetic interactions. Specifically, we will consider the charge radius of S-wave halo nuclei and radiative proton capture into a halo state. Our analysis of finite-range effects also addresses the question of why there are more neutron halos than proton halos in nature.

This manuscript is organized as follows. In Sec. 2, we introduce Halo EFT for S-wave systems and explain how the Coulomb interaction is included into calculations. In Sections 3 and 4, we apply Halo EFT to calculate the charge radius of proton halo nuclei and radiative proton capture, respectively. Results for the excited $1 / 2^{+}$state of Fluorine- 17 are presented in Sec. 5. In particular, we extract the threshold S-factor for radiative proton capture on ${ }^{16} \mathrm{O}$ into ${ }^{17} \mathrm{~F}^{*}$ and the corresponding asymptotic normalization coefficient. To this aim we employ an order-by-order fit to experimental radiative capture data at finite energies and we demonstrate how to quantify theoretical uncertainties within Halo EFT. We address the aforementioned issue of fine tuning in proton halo nuclei in Sec. 6. Finally, we summarize our findings in Sec. 7 .

\section{Theory}

In Halo nuclei, the core and the valence nucleons are the effective degrees of freedom. The Halo EFT Lagrangian can therefore be constructed using only a core and a nucleon field. For proton halos this was first done in Ref. [20]. In order to simplify the inclusion of finite range effects and future extensions to two-proton halos, we use an equivalent approach that introduces an auxiliary halo field $d$ with the quantum numbers of the halo 
nucleus [5], leading to the Lagrangian

$$
\begin{gathered}
\mathcal{L}=\sum_{k=0,1} \psi_{k}^{\dagger}\left[i \mathrm{D}_{0}+\frac{\mathbf{D}^{2}}{2 m_{k}}\right] \psi_{k}+d^{\dagger}\left[\Delta+\nu\left(i \mathrm{D}_{0}+\frac{\mathbf{D}^{2}}{2 M_{\mathrm{tot}}}\right)\right] d \\
-g\left[\psi_{1}^{\dagger} \psi_{0}^{\dagger} d+\text { h.c. }\right]+\ldots
\end{gathered}
$$

The Lagrangian including nucleon and core fields only can be obtained by integrating out the halo field using the classical equations of motion. In Eq. (1), $\psi_{0}$ denotes the proton field with mass $m_{0}$ and $\psi_{1}$ the core field with mass $m_{1}$, while $g$ and $\Delta$ are the low-energy constants of the theory and $M_{\text {tot }}=m_{1}+m_{2}$. The parameter $\nu= \pm 1$ allows for the effective range to be both negative and positive, since we define the coupling $g$ as purely real. ${ }^{1}$ The ellipsis denote operators with more fields and/or additional derivatives. The covariant derivative is $\mathrm{D}_{\mu}=\partial_{\mu}+i e \hat{\mathrm{Q}} A_{\mu}$, where $\hat{\mathrm{Q}}$ is the charge operator and $e>0$ is the elementary electric charge. Low-lying excitations of the core can be included explicitly in the calculation by introducing additional core fields (See, e.g., Refs. [14, 21, 22] for more details). In the calculations below, we do not need to keep track of the proton spin since the core-proton interaction does not change the spin of the proton.

The radiative capture cross section ${ }^{16} \mathrm{O}(p, \gamma){ }^{17} \mathrm{~F}^{*}$ occurs through an initial $\mathrm{P}$-wave state. However, in the Lagrangian (1) only the S-wave part of the strong interaction between the proton and the core has been included. The reason why we do not include the strong $\mathrm{P}$-wave interaction explicitly in the field theory is that all strong initial-state interactions are severely suppressed by the Coulomb repulsion in the energy range that we consider for the capture reaction, $E \leq 2.3 \mathrm{MeV}$. As such we treat the strong P-wave interaction as short-range physics and it is only implicitly included through non-minimal local gauge invariant operators. The initial $\mathrm{P}$-wave state in the radiative capture reaction therefore only interact through the Coulomb potential.

There is a possibility that the strong $\mathrm{P}$-wave interaction is enhanced. If this is the case then it would be of interest to also consider a field theory with the strong P-wave interaction explicitly included. Diagrams with initial strong P-wave interactions would still be suppressed by the Coulomb

\footnotetext{
${ }^{1}$ Note that the kinetic term of the halo field $d$ has the wrong sign for $\nu=-1$ and becomes a ghost field. This introduces no pathologies here since the $d$ field never appears in loops.
} 


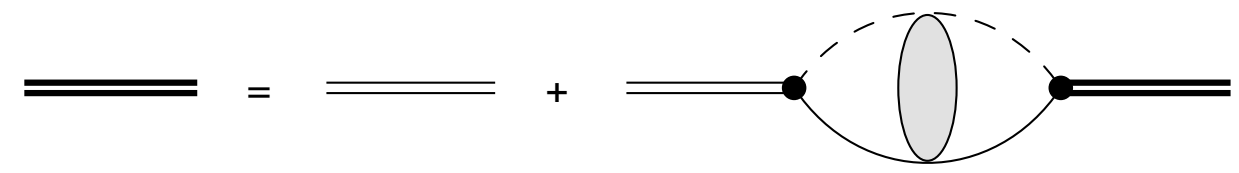

Figure 1: The full halo propagator defined iteratively. The full halo propagator is denoted by the thick double line. The thin double line denotes the bare halo propagator, the dashed single line denotes the core field, the solid single line denotes the proton field and the shaded blob the Coulomb four-point function $\chi$ defined in Fig. 2.

repulsion. However, there would exist additional non-minimal operators at higher orders and therefore the power counting for such a field theory would be different. We will briefly discuss such an operator in Sec. 4.3. Since we have no reason to suspect that the $\mathrm{P}$-wave is enhanced we proceed under the assumption that it scales naturally.

It is important to assign proper scaling dimensions to the fields and operators of the Lagrangian (1) such that we can organize the theory in a power counting. The proton and core fields have the scaling dimension $\psi_{k} \sim 3 / 2$ and the scaling dimension of the halo field is $d \sim 2$ (see for example [14] for a discussion of the scaling dimension of such auxiliary fields). The covariant derivatives scale as $\mathrm{D}_{0} \sim 2$ and $\mathrm{D}_{i} \sim 1$. Note that we do not explicitly count powers of mass factors since mass is not equivalent to energy in nonrelativistic physics.

The Feynman rules for the interactions of the Lagrangian (1) are as follows:

(i) The vertex factor for the strong contact interaction is $-i g$.

(ii) The vertex factors for the $A_{0}$ interaction with the proton, core, and halo field are $-i e,-i e Z_{\mathrm{c}}$ and $-i \nu e\left(Z_{\mathrm{c}}+1\right)$, respectively, where $Z_{\mathrm{c}}$ is the proton number of the core.

(iii) The vertex factors for the interaction of the vector photon, $A_{i}$, with a proton, core, and halo field carrying incoming momentum $\mathbf{p}$ are $-i e \mathbf{p} / m_{0},-i e Z_{\mathrm{c}} \mathbf{p} / m_{1}$ and $-i \nu e\left(Z_{\mathrm{c}}+1\right) \mathbf{p} / M_{\text {tot }}$, respectively.

Note that in our convention the Feynman diagrams are to be read from the right to the left, in the same order as the quantum mechanical matrix elements. 


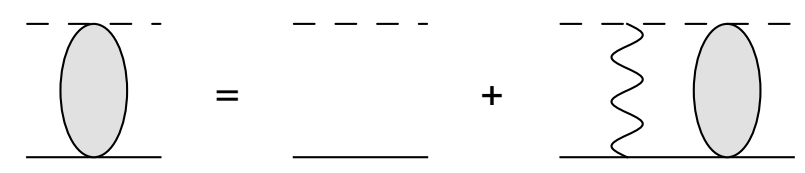

Figure 2: The four-point function $\chi$ defined iteratively. The wiggly line denotes a Coulomb photon exchange. External propagators are amputated. Otherwise, the notation is as in Fig. 1.

From the Lagrangian (1), the one-particle propagator can be deduced to be

$$
i S_{k}\left(p_{0}, \mathbf{p}\right)=i\left[p_{0}-\frac{\mathbf{p}^{2}}{2 m_{k}}+i \varepsilon\right]^{-1} .
$$

For convenience, we will also define the proton-core two-particle propagator

$$
i S_{\mathrm{tot}}\left(p_{0}, \mathbf{p}\right)=i\left[p_{0}-\frac{\mathbf{p}^{2}}{2 m_{\mathrm{R}}}+i \varepsilon\right]^{-1},
$$

where $m_{\mathrm{R}}$ denotes the reduced mass of the proton-core system. In this work, we will only need the halo propagator for a halo field at rest:

$$
i D^{(0)}(E, \mathbf{0}) \equiv i D^{(0)}(E)=\frac{i}{\Delta+\nu(E+i \varepsilon)} .
$$

The corresponding propagator for finite momentum $\mathbf{p}$ can always be obtained by replacing $E \rightarrow E-\mathbf{p}^{2} /\left(2 M_{\text {tot }}\right)$. The power counting for systems interacting through a large scattering length requires that the S-wave interaction is summed up to all orders $[24,25,26]$. The resulting full halo propagator is thus given by the integral equation shown in Fig. 1. For a halo field at rest, we obtain

$$
i D(E)=\frac{i}{\Delta+\nu(E+i \varepsilon)+\Sigma(E)} .
$$

The irreducible self-energy, $\Sigma$, includes strong and Coulomb interactions and will be discussed below.

We include the Coulomb interaction between the core and the valence proton through the full Coulomb Green's function,

$$
\left\langle\mathbf{k}\left|G_{\mathrm{C}}(E)\right| \mathbf{p}\right\rangle=-S_{\mathrm{tot}}(E, \mathbf{k}) \chi(\mathbf{k}, \mathbf{p} ; E) S_{\mathrm{tot}}(E, \mathbf{p}),
$$

where $\mathbf{p}$ and $\mathbf{k}$ are the relative incoming and outgoing momenta and $E$ is the energy. Here, $\chi$ is the momentum-space Coulomb four-point function in the 
center-of-mass frame of the proton and the core, defined recursively in Fig. 2. To distinguish coordinate-space from momentum-space states we will denote the former with round brackets, i.e. |r). The Coulomb Green's function can be expressed via its spectral representation in coordinate space

$$
\left(\mathbf{r}\left|G_{\mathrm{C}}(E)\right| \mathbf{r}^{\prime}\right)=\int \frac{\mathrm{d}^{3} p}{(2 \pi)^{3}} \frac{\psi_{\mathbf{p}}(\mathbf{r}) \psi_{\mathbf{p}}^{*}\left(\mathbf{r}^{\prime}\right)}{E-\mathbf{p}^{2} /\left(2 m_{\mathrm{R}}\right)+i \varepsilon},
$$

where we define the Coulomb wave function through its partial wave expansion

$$
\psi_{\mathbf{p}}(\mathbf{r})=\sum_{l=0}^{\infty}(2 l+1) i^{l} \exp \left(i \sigma_{l}\right) \frac{F_{l}(\eta, \rho)}{\rho} P_{l}(\hat{\mathbf{p}} \cdot \hat{\mathbf{r}}) .
$$

Here we have introduced $\rho=p r$ and the Sommerfeld parameter $\eta=k_{\mathrm{C}} / p$, with the Coulomb momentum $k_{\mathrm{C}}=Z_{\mathrm{c}} \alpha m_{\mathrm{R}}$, where $\alpha$ is the fine structure constant. We have also introduced the pure Coulomb phase shift $\sigma_{l}=$ $\arg \Gamma(l+1+i \eta)$. For the Coulomb functions $F_{l}$ and $G_{l}$, we use the conventions of Ref. [27]. The regular Coulomb function $F_{l}$ can be expressed in terms of the Whittaker M-function according to

$$
F_{l}(\eta, \rho)=A_{l}(\eta) M_{i \eta, l+1 / 2}(2 i \rho)
$$

with the $A_{l}$ defined as

$$
A_{l}(\eta)=\frac{|\Gamma(l+1+i \eta)| \exp [-\pi \eta / 2-i(l+1) \pi / 2]}{2(2 l+1) !} .
$$

We will also need the irregular Coulomb wave function, $G_{l}$, which is given by

$$
G_{l}(\eta, \rho)=i F_{l}(\eta, \rho)+B_{l}(\eta) W_{i \eta, l+1 / 2}(2 i \rho),
$$

where $W$ is the Whittaker $\mathrm{W}$-function and the coefficient $B_{l}$ is defined as

$$
B_{l}(\eta)=\frac{\exp (\pi \eta / 2+i l \pi / 2)}{\arg \Gamma(l+1+i \eta)} .
$$

It is important to note that since we consider both free and bound states the absolute value and the argument of the $\Gamma$-function are given by

$$
|\Gamma(l+1+i \eta)|=\sqrt{\Gamma(l+1+i \eta) \Gamma(l+1-i \eta)}
$$




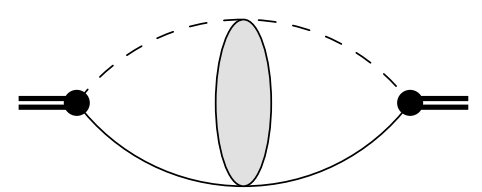

Figure 3: Feynman diagram for the irreducible self energy. The injected four-momentum is $(E, \mathbf{0})$. External legs are amputated. Otherwise, the notation is as in Fig. 1.

and

$$
\arg \Gamma(l+1+i \eta)=\sqrt{\frac{\Gamma(l+1+i \eta)}{\Gamma(l+1-i \eta)}} .
$$

To obtain the fully-dressed two-particle propagator $D$, which includes strong and Coulomb interactions, we calculate the irreducible self-energy shown in Fig. 3. Using Eq. (6), it can be expressed by the momentum-space integral

$$
i \Sigma(E)=-i g^{2} \int \frac{\mathrm{d}^{3} k_{1} \mathrm{~d}^{3} k_{2}}{(2 \pi)^{6}}\left\langle\mathbf{k}_{2}\left|G_{\mathrm{C}}(E)\right| \mathbf{k}_{1}\right\rangle,
$$

which can be written in coordinate space using Fourier transformations:

$$
\begin{aligned}
i \Sigma(E) & =-i g^{2}\left(0\left|G_{\mathrm{C}}(E)\right| 0\right) \\
& =-i g^{2} \int \frac{\mathrm{d}^{3} p}{(2 \pi)^{3}} \frac{\psi_{\mathbf{p}}(0) \psi_{\mathbf{p}}^{*}(0)}{E-\mathbf{p}^{2} /\left(2 m_{\mathrm{R}}\right)+i \varepsilon} .
\end{aligned}
$$

We evaluate this integral using dimensional regularization in the power divergence subtraction (PDS) scheme [25] and the result is [19]

$$
\Sigma(E)=g^{2} \frac{k_{\mathrm{C}} m_{\mathrm{R}}}{\pi} H(\eta)+\Sigma^{\operatorname{div}}
$$

with

$$
H(\eta)=\psi(i \eta)+\frac{1}{2 i \eta}-\log (i \eta)
$$

where $\psi(z)=\Gamma^{\prime}(z) / \Gamma(z)$ is the logarithmic derivative of the Gamma function. The divergent part is given by

$$
\Sigma^{\operatorname{div}}=-\frac{g^{2} k_{\mathrm{C}} m_{\mathrm{R}}}{\pi}\left[\frac{1}{3-d}+\log \left(\frac{\sqrt{\pi} \mu}{2 k_{\mathrm{C}}}\right)+1-\frac{3 C_{\mathrm{E}}}{2}\right]+\frac{g^{2} m_{\mathrm{R}} \mu}{2 \pi},
$$

with the renormalization scale $\mu$. Note that $\Sigma^{\text {div }}$ is independent of energy and therefore will vanish when we take the energy derivative of the irreducible self-energy to arrive at the LSZ residue below. 


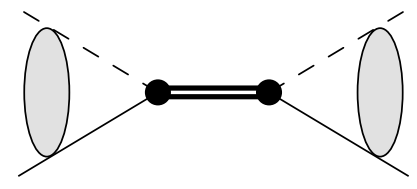

Figure 4: Scattering amplitude for elastic proton-core scattering. The notation is as in Fig. 1.

\subsection{Renormalization}

Expressions for EFT low-energy constants such as $g$ and $\Delta$ defined above are frequently obtained by matching them to elastic scattering observables. The amplitude for elastic proton-core scattering is obtained from the diagram shown in Fig. 4. It evaluates to the S-wave t-matrix

$$
i T_{0}(E)=i g^{2} \exp \left(2 i \sigma_{0}\right) C_{\eta}^{2} D(E),
$$

with the Gamow-Sommerfeld factor $C_{\eta}^{2}=\Gamma(1+i \eta) \Gamma(1-i \eta)$.

In the absence of the Coulomb interaction, the t-matrix is usually expressed in terms of effective range parameters. However, it is not possible to separate the strong interaction from the Coulomb interaction in a model-independent way. Therefore one uses the so-called Coulomb-modified effective range expansion (ERE) [28] to relate the phase shifts to redefined effective range parameters. The $t$-matrix is then written

$$
T_{0}(E)=-\frac{2 \pi}{m_{\mathrm{R}}} \frac{C_{\eta}^{2} \exp \left(2 i \sigma_{0}\right)}{k C_{\eta}^{2}\left(\cot \delta_{0}-i\right)},
$$

where the total phase shift is given by $\sigma_{0}+\delta_{0}$. The S-wave Coulomb-modified ERE is

$$
k C_{\eta}^{2}\left(\cot \delta_{0}-i\right)+2 k_{\mathrm{C}} H(\eta)=-\frac{1}{a_{0}}+\frac{1}{2} r_{0} k^{2}+\ldots,
$$

where $a_{0}$ and $r_{0}$ are the Coulomb-modified scattering length and effective range, respectively. One should note that the imaginary part of $2 k_{\mathrm{C}} H(\eta)$ exactly cancels $-i k C_{\eta}^{2}$.

Comparing Eqs. (20) and (21), order by order in the momentum $k$, we can express the scattering length and the effective range in terms of the 
low-energy coupling constants $g$ and $\Delta$

$$
\begin{aligned}
\frac{1}{a_{0}} & =\frac{2 \pi}{g^{2} m_{\mathrm{R}}}\left(\Delta+\Sigma^{\mathrm{div}}\right), \\
r_{0} & =-\frac{2 \pi \nu}{g^{2} m_{\mathrm{R}}^{2}} .
\end{aligned}
$$

Equation (23) shows how the low-energy constant $\Delta$ absorbs the divergent part of the irreducible self-energy $\Sigma^{\text {div }}$. Furthermore, at LO we effectively take $\nu$ to zero. The two parameters $g$ and $\Delta$ are then not independent.

The residue at the bound state, $E=-B$, of the full halo propagator defines the wavefunction renormalization, or the LSZ residue, and is therefore required for the calculation of bound-state observables. It is given by

$$
\mathcal{Z}=\left.\left[\frac{\mathrm{d}\left(D^{-1}\right)}{\mathrm{d} E}\right]^{-1}\right|_{E=-B}=\frac{1}{\nu+\Sigma^{\prime}(-B)}
$$

In terms of the effective range, we write this as

$$
\mathcal{Z}=\frac{6 \pi k_{\mathrm{C}}}{g^{2} m_{\mathrm{R}}^{2}} \frac{1}{\tilde{H}\left(\gamma, k_{\mathrm{C}}\right)-3 k_{\mathrm{C}} r_{0}},
$$

using the matching condition in Eq. (24). In writing Eq. (26), we have defined the function

$$
\tilde{H}\left(\gamma, k_{\mathrm{C}}\right)=\left.\frac{6 k_{\mathrm{C}}^{2}}{m_{\mathrm{R}}} \frac{\mathrm{d}}{\mathrm{d} E} H(\eta)\right|_{E=-B},
$$

with the binding momentum $\gamma=\sqrt{2 m_{\mathrm{R}} B}$. The expression Eq. (26) is valid at next-to-leading order (NLO), that is it includes the effective-range correction. At leading order ( $\mathrm{LO})$ the wavefunction renormalization is given by the simpler expression

$$
\begin{aligned}
\mathcal{Z}_{\mathrm{LO}} & =\frac{1}{\Sigma^{\prime}(-B)} \\
& =\frac{6 \pi k_{\mathrm{C}}}{g^{2} m_{\mathrm{R}}^{2}} \frac{1}{\tilde{H}\left(\gamma, k_{\mathrm{C}}\right)}
\end{aligned}
$$

that is Eq. (26) with $r_{0}$ set to zero. Note that the factor of $g^{2}$ in the denominator of the wavefunction renormalization will always cancel with a corresponding factor in an unrenormalized matrix element. 
If the ratio between the binding momentum and Coulomb momentum $\gamma / k_{\mathrm{C}}$ is small we can expand the function $\tilde{H}\left(\gamma, k_{\mathrm{C}}\right)$ according to

$$
\tilde{H}\left(\gamma, k_{\mathrm{C}}\right)=1-\frac{\gamma^{2}}{5 k_{\mathrm{C}}^{2}}+\frac{\gamma^{4}}{7 k_{\mathrm{C}}^{4}}+\ldots .
$$

Thus, for systems where the separation $\gamma \ll k_{\mathrm{C}}$ is fulfilled, we can use $\tilde{H}\left(\gamma, k_{\mathrm{C}}\right) \rightarrow 1$ in all expressions. (See Ref. [23] for a similar expansion of $H(\eta)$.)

In this paper, we will fix the LSZ residue $\mathcal{Z}$ to a calculated or measured asymptotic normalization coefficient (ANC), $A$, or to experimental radiative capture data. The ANC is defined as the coefficient in the asymptotic bound state wavefunction

$$
w_{l}(r)=A W_{-i \eta, l+1 / 2}(2 \gamma r),
$$

where $W$ is the Whittaker-W function. The LSZ residue is related to the ANC according to

$$
\mathcal{Z}=\frac{\pi}{g^{2} m_{\mathrm{R}}^{2}\left[\Gamma\left(1+k_{\mathrm{C}} / \gamma\right)\right]^{2}} A^{2} .
$$

At LO, the wavefunction renormalization is determined solely by $\gamma$ and $k_{\mathrm{C}}$, as can be seen in Eq. (29), and as such this could in principle be used to predict the LO ANC as

$$
A_{\mathrm{LO}}=\sqrt{\frac{6 k_{\mathrm{C}}}{\tilde{H}\left(\gamma, k_{\mathrm{C}}\right)}} \Gamma\left(1+k_{\mathrm{C}} / \gamma\right) .
$$

At NLO we are left with one undetermined parameter, $r_{0}$, in the LSZ residue (26). Thus, at orders beyond $\mathrm{LO}$ we can use an ANC as input to fix $\mathcal{Z}$ and to predict the effective-range parameters. We will therefore define our NLO wavefunction renormalization in terms of the matching to the ANC, Eq. (32). The ratio to the LO residue is then obtained as

$$
\frac{\mathcal{Z}}{\mathcal{Z}_{\mathrm{LO}}}=\frac{\tilde{H}\left(\gamma, k_{\mathrm{C}}\right) A^{2}}{6 k_{\mathrm{C}}\left[\Gamma\left(1+k_{\mathrm{C}} / \gamma\right)\right]^{2}} .
$$

It is important to note that the LSZ-residue and ANC receive contributions from all ERE parameters. As such the expression (26) is only valid at NLO. However, in this paper we fit the exact ANC to data and make predictions of the S-factor and the charge form factor without extracting the ERE parameters. The matching (32) is therefore valid at any order in the power counting 
and there is no EFT error due to the non-inclusion of higher-order contact interaction.

For completeness, we also provide expressions for the effective range and the scattering length, up to corrections of higher-order ERE parameters. For a given one-proton separation energy, the ANC determines the Coulombmodified effective range

$$
r_{0}=\frac{1}{3 k_{\mathrm{C}}}\left[\tilde{H}\left(\gamma, k_{\mathrm{C}}\right)-\frac{6 k_{\mathrm{C}} \Gamma\left(1+k_{\mathrm{C}} / \gamma\right)^{2}}{A^{2}}\right]
$$

combining Eqs. (26) and (32). The Coulomb-modified scattering length is then obtained from the pole position of the t-matrix (21), that is

$$
a_{0}=-\frac{2}{4 k_{\mathrm{C}} H\left(-i k_{\mathrm{C}} / \gamma\right)+\gamma^{2} r_{0}}
$$

These S-wave effective-range parameter predictions are accurate up to corrections of the shape parameter in the ERE. It is therefore important to note that if the ERE parameters are to be constrained to high accuracy by the ANC, then one would need to derive the expressions (35) and (36) to higher orders. Vice versa, if the ANC is to be predicted accurately from elastic scattering data, then the expression (26) needs to be improved on with dependencies of additional ERE parameters.

\section{Charge form factor}

Information on the electromagnetic structure of an object can be obtained through elastic electron scattering. The elastic scattering amplitude can then be expressed in terms of the electric and magnetic form factors. Here we will focus on the electric charge form factor of an S-wave halo nucleus. The charge form factor, $F_{\mathrm{C}}$, and charge radius, $r_{\mathrm{C}}$, are defined by

$$
\begin{aligned}
F_{\mathrm{C}}(Q) & =\frac{1}{e\left(Z_{\mathrm{c}}+1\right)}\left\langle\mathbf{p}^{\prime}\left|J_{\mathrm{EM}}^{0}\right| \mathbf{p}\right\rangle \\
& =1-\frac{r_{\mathrm{C}}^{2}}{6} Q^{2}+\ldots
\end{aligned}
$$

where $\mathbf{p}\left(\mathbf{p}^{\prime}\right)$ is the incoming (outgoing) momentum of the halo field, $\mathbf{Q}=$ $\mathbf{p}^{\prime}-\mathbf{p}$ is the momentum transfer, and $J_{\mathrm{EM}}^{\mu}$ is the electromagnetic current. We 


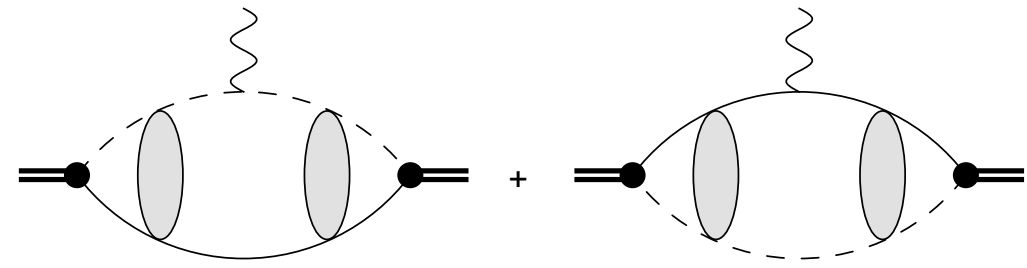

Figure 5: The diagrams for the charge form factor at LO. The notation is as in Fig. 1.

evaluate this observable in the Breit frame, where no energy is transferred such that the photon four-momentum is $(0, \mathbf{Q})$.

In this paper, we calculate the charge form factor to NLO, by evaluating the relevant diagrams to this order. At LO there are two loop diagrams, $\Gamma_{\mathrm{LO}}(Q)$, and at NLO a constant tree-level diagram, $\Gamma_{\mathrm{NLO}}$, enters. We derive and evaluate these diagrams below. The charge form factor is then given by the sum of diagrams

$$
F_{\mathrm{C}}(Q)=\frac{1}{e\left(Z_{\mathrm{c}}+1\right)} \mathcal{Z}\left[\Gamma_{\mathrm{LO}}(Q)+\Gamma_{\mathrm{NLO}}(Q)+\ldots\right] .
$$

\subsection{Leading order}

At leading order, we have to consider the diagrams shown in Fig. 5. There, the photon couples to the single-particle lines only, that is through an operator $\psi_{k}^{\dagger} A_{0} \psi_{k}$ of dimension 5. We choose incoming and outgoing total four-momenta as $(E,-\mathbf{Q} / 2)$ and $(E, \mathbf{Q} / 2)$, respectively. The resulting amplitude in momentum space is given by

$$
\begin{aligned}
i \Gamma_{\mathrm{LO}}(Q)= & i g^{2} e Z_{\mathrm{c}} \int \frac{\mathrm{d}^{4} k_{1} \mathrm{~d}^{4} k_{2} \mathrm{~d}^{4} k_{3}}{(2 \pi)^{12}} i S_{0}\left(k_{30}, \mathbf{k}_{3}\right) \\
& \times i S_{1}\left(E-k_{30},-\mathbf{k}_{3}+\mathbf{Q} / 2\right) i \chi\left(\mathbf{k}_{3}-f \mathbf{Q} / 2, \mathbf{k}_{2}-f \mathbf{Q} / 2,-B\right) \\
& \times i S_{0}\left(k_{20}, \mathbf{k}_{2}\right) i S_{1}\left(E-k_{20},-\mathbf{k}_{2}+\mathbf{Q} / 2\right) \\
& \times i S_{1}\left(E-k_{20},-\mathbf{k}_{2}-\mathbf{Q} / 2\right) i \chi\left(\mathbf{k}_{2}+f \mathbf{Q} / 2, \mathbf{k}_{1}+f \mathbf{Q} / 2,-B\right) \\
& \times i S_{0}\left(k_{10}, \mathbf{k}_{1}\right) i S_{1}\left(E-k_{10},-\mathbf{k}_{1}-\mathbf{Q} / 2\right) \\
& +\left[(f \rightarrow 1-f),\left(Z_{\mathrm{c}} \rightarrow 1\right),\left(S_{0} \leftrightarrow S_{1}\right)\right]
\end{aligned}
$$

where $f=m_{0} / M_{\text {tot }}$ is a mass ratio introduced for convenience. The last row makes sure that the photon also couples to the proton. Note that the total energy $E$ is given by $E=-B+Q^{2} /\left(8 M_{\text {tot }}\right)$, using energy conservation. 
We evaluate the energy integrals in Eq. (40) using the residue theorem, noting that the poles are located at $k_{n 0}=-i \varepsilon+\mathbf{k}_{n}^{2} /\left(2 m_{0}\right)$ for $n=1,2,3$. The result can be written using two-body propagators, defined in Eq. (3), as

$$
\begin{aligned}
i \Gamma_{\mathrm{LO}}(Q)= & -i g^{2} e Z_{\mathrm{c}} \int \frac{\mathrm{d}^{3} k_{1} \mathrm{~d}^{3} k_{2} \mathrm{~d}^{3} k_{3}}{(2 \pi)^{9}} S_{\mathrm{tot}}\left(-B, \mathbf{k}_{3}\right) \\
& \times \chi\left(\mathbf{k}_{3}, \mathbf{k}_{2}-f \mathbf{Q} / 2,-B\right) S_{\mathrm{tot}}\left(-B, \mathbf{k}_{2}-f \mathbf{Q} / 2\right) \\
& \times S_{\mathrm{tot}}\left(-B, \mathbf{k}_{2}+f \mathbf{Q} / 2\right) \chi\left(\mathbf{k}_{2}+f \mathbf{Q} / 2, \mathbf{k}_{1},-B\right) \\
& \times S_{\text {tot }}\left(-B, \mathbf{k}_{1}\right) \\
& +\left[(f \rightarrow 1-f),\left(Z_{\mathrm{c}} \rightarrow 1\right)\right],
\end{aligned}
$$

and this expression can be simplified using the Coulomb Green's function in Eq. (6), to replace the two-body propagators $S_{\text {tot }}$ and the four-point function $\chi$. This leads to

$$
\begin{aligned}
i \Gamma_{\mathrm{LO}}(Q)= & -i g^{2} e Z_{\mathrm{c}} \int \frac{\mathrm{d}^{3} k_{1} \mathrm{~d}^{3} k_{2} \mathrm{~d}^{3} k_{3}}{(2 \pi)^{9}}\left\langle\mathbf{k}_{3}\left|G_{\mathrm{C}}(-B)\right| \mathbf{k}_{2}-f \mathbf{Q} / 2\right\rangle \\
& \times\left\langle\mathbf{k}_{2}+f \mathbf{Q} / 2\left|G_{\mathrm{C}}(-B)\right| \mathbf{k}_{1}\right\rangle \\
& +\left[(f \rightarrow 1-f),\left(Z_{\mathrm{c}} \rightarrow 1\right)\right] .
\end{aligned}
$$

By performing a Fourier transform on each of the momentum-space bras and kets, we arrive at the coordinate-space integral

$$
\begin{aligned}
i \Gamma_{\mathrm{LO}}(Q)= & -i g^{2} e Z_{\mathrm{c}} \int \frac{\mathrm{d}^{3} r}{(2 \pi)^{3}} \\
& \times\left(0\left|G_{\mathrm{C}}(-B)\right| \mathbf{r}\right) \exp (i f \mathbf{Q} \cdot \mathbf{r})\left(\mathbf{r}\left|G_{\mathrm{C}}(-B)\right| 0\right) \\
& +\left[(f \rightarrow 1-f),\left(Z_{\mathrm{c}} \rightarrow 1\right)\right] .
\end{aligned}
$$

This integral is much more convenient to use than the rather involved momentum-space integral in Eq. (42). In the integral (43) the diagram in Fig. 5 is also visualized better. It consists of two Coulomb Green's functions, that propagate the fields from separation zero to $\mathbf{r}$ and back from separation $\mathbf{r}$ to zero, respectively, and the current operator in between the propagators. Since the Coulomb Green's functions have one end at zero separation, only the S-wave part of these will contribute. Therefore we expand the Coulomb Green's function in partial waves

$$
\left(\mathbf{r}^{\prime}\left|G_{\mathrm{C}}(E)\right| \mathbf{r}\right)=\sum_{l=0}^{\infty}(2 l+1) G_{\mathrm{C}}^{(l)}\left(E ; r^{\prime}, r\right) P_{l}\left(\hat{\mathbf{r}}^{\prime} \cdot \hat{\mathbf{r}}\right),
$$


with the bound-state partial-wave projected Coulomb Green's function

$$
G_{\mathrm{C}}^{(l)}\left(-B ; r^{\prime}, r\right)=-i \frac{m_{\mathrm{R}} \gamma}{2 \pi} \frac{F_{l}\left(\eta, \rho^{\prime}\right)\left[i F_{l}(\eta, \rho)+G_{l}(\eta, \rho)\right]}{\rho^{\prime} \rho} .
$$

We can now write the Green's function as (see Appendix A for details)

$$
\begin{aligned}
\left(0\left|G_{\mathrm{C}}(-B)\right| \mathbf{r}\right) & =G_{\mathrm{C}}^{(0)}(-B ; 0, r) \\
& =-\frac{m_{\mathrm{R}} \Gamma\left(1+k_{\mathrm{C}} / \gamma\right)}{2 \pi} \frac{W_{-k_{\mathrm{C}} / \gamma, 1 / 2}(2 \gamma r)}{r} .
\end{aligned}
$$

As such, the integral (43) can be written as

$$
\begin{aligned}
i \Gamma_{\mathrm{LO}}(Q)= & -i \frac{g^{2} e Z_{\mathrm{c}} m_{\mathrm{R}}^{2}}{8 \pi^{4}} \Gamma\left(1+k_{\mathrm{C}} / \gamma\right)^{2} \int \mathrm{d} r j_{0}(f Q r) W_{-k_{\mathrm{C}} / \gamma, 1 / 2}(2 \gamma r)^{2} \\
& +\left[(f \rightarrow 1-f),\left(Z_{\mathrm{c}} \rightarrow 1\right)\right]
\end{aligned}
$$

This integral can be evaluated numerically in a straightforward way. The LO charge form factor can now be calculated through

$$
\left.F_{\mathrm{C}}(Q)\right|_{\mathrm{LO}}=\frac{1}{e\left(Z_{\mathrm{c}}+1\right)} \mathcal{Z}_{\mathrm{LO}} \Gamma_{\mathrm{LO}}(Q)
$$

and the LO charge radius is given in terms of the loop-integral $\Gamma_{\mathrm{LO}}(Q)$ and the wavefunction renormalization $\mathcal{Z}_{\mathrm{LO}}$, according to

$$
\left.r_{\mathrm{C}}^{2}\right|_{\mathrm{LO}}=-\left.\frac{3 \mathcal{Z}_{\mathrm{LO}}}{e\left(Z_{\mathrm{c}}+1\right)} \frac{\mathrm{d}^{2}}{\mathrm{~d}^{2} Q} \Gamma_{\mathrm{LO}}(Q)\right|_{Q=0} .
$$

We will now show that the charge form factor is normalized correctly to 1 at $Q=0$. Starting from the coordinate-space integral (43) at $Q=0$ and the spectral representation of the Coulomb Green's function

$$
\left(0\left|G_{\mathrm{C}}(E)\right| \mathbf{r}\right)=\int \frac{\mathrm{d}^{3} p}{(2 \pi)^{3}} \frac{\psi_{\mathbf{p}}(0) \psi_{\mathbf{p}}^{*}(\mathbf{r})}{E-\mathbf{p}^{2} /\left(2 m_{\mathrm{R}}\right)+i \varepsilon},
$$

we find that

$$
\begin{aligned}
\Gamma_{\mathrm{LO}}(0) & =-g^{2} e\left(Z_{\mathrm{c}}+1\right) \int \mathrm{d}^{3} r\left|\left(0\left|G_{\mathrm{C}}(-B)\right| r\right)\right|^{2} \\
& =-g^{2} e\left(Z_{\mathrm{c}}+1\right) \int \frac{\mathrm{d}^{3} p}{(2 \pi)^{3}} \frac{\psi_{\mathbf{p}}(0) \psi_{\mathbf{p}}^{*}(0)}{\left(E-\mathbf{p}^{2} /\left(2 m_{\mathrm{R}}\right)+i \varepsilon\right)^{2}} \\
& =e\left(Z_{\mathrm{c}}+1\right) \Sigma^{\prime}(-B) .
\end{aligned}
$$




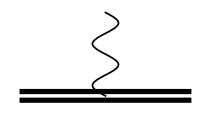

Figure 6: The diagram for the charge form factor at NLO. The notation is as in Fig. 1.

In the first step above, a Dirac delta was used, coming from the integration over the orthonormal Coulomb wavefunctions

$$
\int \mathrm{d}^{3} r \psi_{\mathbf{p}}^{*}(\mathbf{r}) \psi_{\mathbf{p}^{\prime}}(\mathbf{r})=(2 \pi)^{3} \delta^{(3)}\left(\mathbf{p}-\mathbf{p}^{\prime}\right) .
$$

The correct normalization of the LO charge form factor now follows, combining Eqs. (48), (51) and (28).

\subsection{Next-to-leading order}

The contributions that enter at NLO are that the full LSZ residue (25), or (32), is to be used and that the NLO tree-level diagram, given in Fig. 6, enters, through the operator $d^{\dagger} A_{0} d$ of dimension 6 . This diagram is simply given by the Feynman rule for an $A_{0}$ photon coupling to the dicluster field

$$
i \Gamma_{\mathrm{NLO}}=i \nu e\left(Z_{\mathrm{c}}+1\right) \text {. }
$$

Note that the NLO correction diagram (53) is independent of the momentum transfer $Q$. These contributions come with an effective-range correction.

The NLO charge form factor is given by the sum of the diagrams up to NLO, according to Eq. (39). It is clear that the normalization of the charge form factor at $Q=0$ is still correct, using Eq. (51) together with the NLO LSZ residue (25), the NLO diagram (53) and the formula (39). The charge radius is then given by the order $Q^{2}$ part of the LO loop-integral (47) together with the full wavefunction renormalization (32). The resulting NLO charge radius result is

$$
r_{\mathrm{C}}^{2}=-\left.\frac{3 \mathcal{Z}}{e\left(Z_{\mathrm{c}}+1\right)} \frac{\mathrm{d}^{2}}{\mathrm{~d}^{2} Q} \Gamma_{\mathrm{LO}}(Q)\right|_{Q=0} .
$$

It is evident that the NLO charge radius squared is a factor

$$
\frac{\mathcal{Z}}{\mathcal{Z}_{\mathrm{LO}}}=\frac{\tilde{H}\left(\gamma, k_{\mathrm{C}}\right) A^{2}}{6 k_{\mathrm{C}} \Gamma\left(1+k_{\mathrm{C}} / \gamma\right)^{2}} .
$$

larger (or smaller) than the LO result. 
At higher orders there are three types of corrections. Firstly, there are local short-range operators $\psi_{k}^{\dagger}\left[\nabla^{2} A_{0}-\partial_{0}(\nabla \cdot \mathbf{A})\right] \psi_{k}$, of dimension 7 , which enter with finite-size contributions of the core and proton fields. Secondly, there is a local short-range operator $d^{\dagger}\left[\nabla^{2} A_{0}-\partial_{0}(\nabla \cdot \mathbf{A})\right] d$, of dimension 8 , that comes in with an undetermined short-range parameter. Thirdly, there are photon couplings due to the minimal substitution of derivatives in higherorder contact interactions, with the leading $\mathrm{N}^{3} \mathrm{LO}$ contribution coming from the shape-parameter in the ERE.

\section{Radiative capture}

In this section we consider radiative capture of a proton into a halo state. By including range corrections, we are able to compute the cross section for this process to high orders in the Halo EFT expansion. We use quantum numbers relevant for the capture process ${ }^{16} \mathrm{O}(\mathrm{p}, \gamma){ }^{17} \mathrm{~F}^{*}$, which require that the incoming particle pair has relative angular momentum $l \geq 1$. Specifically, we will consider the E1 capture through an incoming $\mathrm{P}$-wave.

The differential cross section for radiative capture of non-relativistic particles is

$$
\frac{\mathrm{d} \sigma}{\mathrm{d} \Omega}=\frac{m_{\mathrm{R}} \omega}{8 \pi^{2} p} \sum_{i}\left|\epsilon_{i} \cdot \mathcal{A}\right|^{2},
$$

where $\omega$ is the energy of the outgoing photon, $p$ is the relative momentum of the incoming particle pair and $\epsilon_{i}$ are the photon polarization vectors. The vector amplitude $\mathcal{A}$ is for the capture process with a vector photon $A_{i}$ being emitted. Note that we are working in Coulomb gauge, where the relation

$$
\epsilon_{i} \cdot \mathrm{Q}=0 \text {, }
$$

for a real photon with momentum $\mathbf{Q}$, is fulfilled.

We present our results in terms of the astrophysical S-factor, which is defined as

$$
S(E)=E \exp (2 \pi \eta) \sigma_{\mathrm{tot}}(E)
$$

with the incoming center-of-mass energy $E$ and the total cross section $\sigma_{\text {tot }}$.

From the Lagrangian (1), we can write down three classes of diagrams for the radiative capture process. Two of these are identically zero since the incoming particle pair is in a relative S-wave. For completeness we will show this explicitly below. The remaining diagram has contributions from partial waves $l \geq 1$, but since the $\mathrm{P}$-wave dominates at low energies we neglect all other partial waves. 


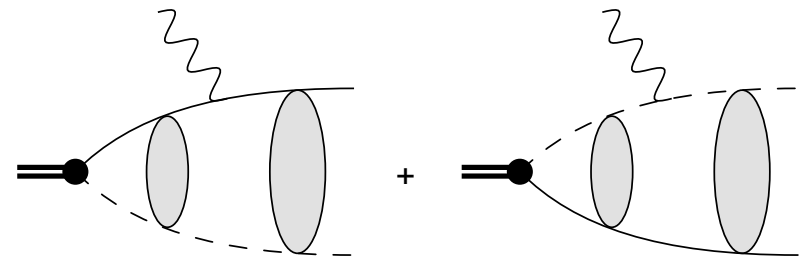

Figure 7: The diagrams for radiative capture at LO. The notation is as in Fig. 1 and time flows from right to left.

\subsection{Leading order}

At leading order, we only consider diagrams where the photon couples to one of the single-particle lines, that is through an operator $\psi_{k}^{\dagger} \frac{i e \hat{\mathrm{Q}} A_{i} \nabla_{i}}{m_{k}} \psi_{k}$ of dimension 5. The two radiative capture diagrams of interest are shown in Fig. 7. These diagrams are to be read from the right to the left, that is with the incoming proton-core pair on the right-hand side and the outgoing bound state to the left. We will write them using the pure Coulomb t-matrix $T_{\mathrm{C}}$ [29]. The four-point function $\chi$ defined in Fig. 2 is directly proportional to the Coulomb Green's function $G_{C}$ and receives contributions from the Coulomb t-matrix $T_{C}$ as well as from the free propagation of the core-proton system (cf. Fig. 2). In the center-of-mass of the halo and the radiated photon, the momentum space diagrams from Fig. 7 are given by

$$
\begin{aligned}
i \mathcal{A}= & -i g \sqrt{\mathcal{Z}_{\mathrm{LO}}} \int \frac{\mathrm{d}^{4} k_{2}}{(2 \pi)^{4}} i S_{0}\left(k_{20}, \mathbf{k}_{2}\right) i S_{1}\left(E-k_{20}-\omega,-\mathbf{k}_{2}-\mathbf{Q}\right) \\
& \times\left[i \chi\left(\mathbf{k}_{2}+f \mathbf{Q}, \mathbf{p}+f \mathbf{Q},-B\right) i S_{1}\left(\mathbf{p}^{2} /\left(2 m_{1}\right)-\omega,-\mathbf{p}-\mathbf{Q}\right)\right. \\
& \times\left(-i e Z_{\mathrm{c}}(-\mathbf{p}) / m_{1}\right) \\
& +\int \frac{\mathrm{d}^{4} k_{1}}{(2 \pi)^{4}} i \chi\left(\mathbf{k}_{2}+f \mathbf{Q}, \mathbf{k}_{1}+f \mathbf{Q},-B\right) i S_{1}\left(E-k_{10}-\omega,-\mathbf{k}_{1}-\mathbf{Q}\right) \\
& \times\left(-i e Z_{\mathrm{c}}\left(-\mathbf{k}_{1}\right) / m_{1}\right) \\
& \left.\times i S_{1}\left(E-k_{10},-\mathbf{k}_{1}\right) i S_{0}\left(k_{10}, \mathbf{k}_{1}\right)\left(-i T_{\mathrm{C}}\left(\mathbf{k}_{1}, \mathbf{p}\right)\right)\right] \\
& -\left[(f \rightarrow 1-f),\left(Z_{\mathrm{c}} \rightarrow 1\right),\left(m_{0} \leftrightarrow m_{1}\right),\left(S_{0} \leftrightarrow S_{1}\right)\right],
\end{aligned}
$$

where we have separated the contributions from the Coulomb t-matrix $T_{C}$ and the free propagation of the core-proton system in the initial state. The total energy flowing through the diagram is $E=-B+\omega+\omega^{2} /\left(2 M_{\text {tot }}\right)$ 
and the factor $\sqrt{\mathcal{Z}_{\mathrm{LO}}}$ is from the wavefunction renormalization of the halo. Performing the $k_{n 0}$ residue integrals and introducing a convenient Dirac delta, the integral (58) can be written as

$$
\begin{aligned}
i \mathcal{A}= & -i g \sqrt{\mathcal{Z}_{\mathrm{LO}}} \frac{e Z_{\mathrm{c}} f}{m_{\mathrm{R}}} \int \frac{\mathrm{d}^{3} k_{1} \mathrm{~d}^{3} k_{2}}{(2 \pi)^{6}} S_{\mathrm{tot}}\left(-B, \mathbf{k}_{2}+f \mathbf{Q}\right) \chi\left(\mathbf{k}_{2}+f \mathbf{Q}, \mathbf{k}_{1}+f \mathbf{Q},-B\right) \\
& \times S_{\mathrm{tot}}\left(-B, \mathbf{k}_{1}+f \mathbf{Q}\right) \mathbf{k}_{1} \\
& \times\left[\delta^{3}\left(\mathbf{p}-\mathbf{k}_{1}\right)+\left[E-\mathbf{k}_{1}^{2} /\left(2 m_{\mathrm{R}}\right)\right]^{-1} T_{\mathrm{C}}\left(\mathbf{k}_{1}, \mathbf{p}\right)\right] \\
& -\left[(f \rightarrow 1-f),\left(Z_{\mathrm{c}} \rightarrow 1\right)\right] .
\end{aligned}
$$

Then, using the Lippmann-Schwinger relation [29]

$$
\psi_{\mathbf{p}}\left(\mathbf{k}_{1}\right)=\delta^{3}\left(\mathbf{k}_{1}-\mathbf{p}\right)+\left[E-\mathbf{k}_{1}^{2} /\left(2 m_{\mathrm{R}}\right)\right]^{-1} T_{\mathrm{C}}\left(\mathbf{k}_{1}, \mathbf{p}\right)
$$

and replacing the four-point function $\chi$ with Eq. (6) the integral (59) can be expressed in a simpler fashion:

$$
\begin{aligned}
i \mathcal{A}= & -i g \sqrt{\mathcal{Z}_{\mathrm{LO}}} \frac{e Z_{\mathrm{c}} f}{m_{\mathrm{R}}} \int \frac{\mathrm{d}^{3} k_{1} \mathrm{~d}^{3} k_{2}}{(2 \pi)^{6}}\left\langle\mathbf{k}_{2}\left|G_{\mathrm{C}}(-B)\right| \mathbf{k}_{1}+f \mathbf{Q}\right\rangle \mathbf{k}_{1} \psi_{\mathbf{p}}\left(\mathbf{k}_{1}\right) \\
& -\left[(f \rightarrow 1-f),\left(Z_{\mathrm{c}} \rightarrow 1\right)\right]
\end{aligned}
$$

Performing Fourier transforms and using $\mathbf{k}_{1} \exp \left(i \mathbf{k}_{1} \cdot \mathbf{r}_{2}\right)=-i \nabla_{2} \exp \left(i \mathbf{k}_{1} \cdot \mathbf{r}_{2}\right)$ we can write the integral (61) as

$$
\begin{aligned}
i \mathcal{A}= & -g \sqrt{\mathcal{Z}_{\mathrm{LO}}} \frac{e Z_{\mathrm{c}} f}{m_{\mathrm{R}}} \int \mathrm{d}^{3} r_{1} \mathrm{~d}^{3} r_{2} G_{\mathrm{C}}^{(0)}\left(-B ; 0, p r_{1}\right) \psi_{\mathbf{p}}\left(\mathbf{r}_{2}\right) \\
& \times \exp \left(-i f \mathbf{Q} \cdot \mathbf{r}_{1}\right) \nabla_{2} \delta^{(3)}\left(\mathbf{r}_{2}-\mathbf{r}_{1}\right) \\
& -\left[(f \rightarrow 1-f),\left(Z_{\mathrm{c}} \rightarrow 1\right)\right] .
\end{aligned}
$$

The resulting integral can now be expressed as

$$
\begin{aligned}
i \mathcal{A}= & g \sqrt{\mathcal{Z}_{\mathrm{LO}}} \frac{e Z_{\mathrm{c}} f}{m_{\mathrm{R}}} \int \mathrm{d}^{3} r G_{\mathrm{C}}^{(0)}(-B ; 0, p r) \exp (-i f \omega r \cos \theta)\left(\nabla \psi_{\mathbf{p}}(\mathbf{r})\right) \\
& -\left[(f \rightarrow 1-f),\left(Z_{\mathrm{c}} \rightarrow 1\right)\right] .
\end{aligned}
$$




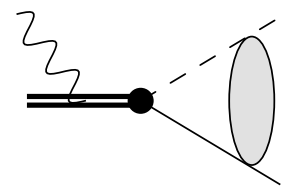

Figure 8: The first of the two vanishing classes of capture diagrams at LO. The notation is as in Fig. 1 and time flows from right to left.

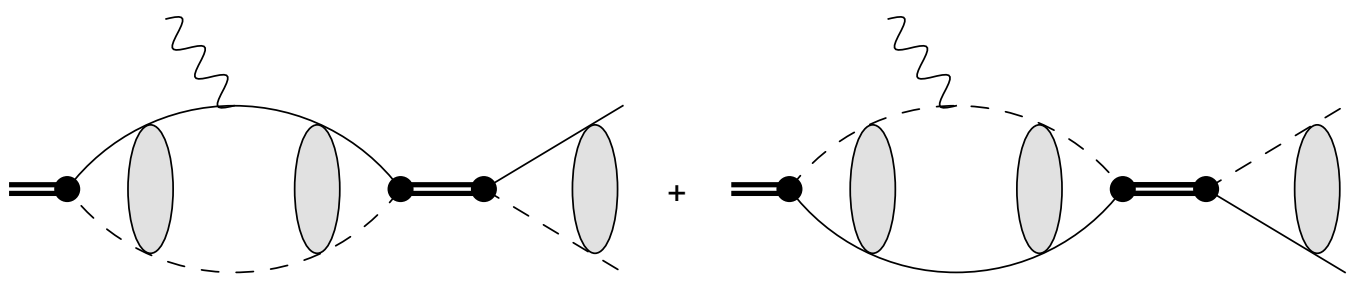

Figure 9: The second of the two vanishing classes of capture diagrams at LO. The notation is as in Fig. 1 and time flows from right to left.

Summing over all polarizations and doing the angular integration, the amplitude squared in Eq. (63) evaluates to

$$
\begin{aligned}
\sum_{i}\left|\epsilon_{i} \cdot \mathcal{A}\right|^{2}= & \mid \sqrt{\mathcal{Z}} \sin \theta(\cos \phi+\sin \phi) \frac{4 \pi g e Z_{\mathrm{c}} f \exp \left(i \sigma_{1}\right)}{m_{\mathrm{R}} p} \\
& \times \int \mathrm{d} r G_{\mathrm{C}}^{(0)}(-B ; 0, \rho) j_{0}(f \omega r) \frac{\partial}{\partial r}\left[r F_{1}\left(k_{\mathrm{C}} / p, p r\right)\right] \\
& -\left.\left[(f \rightarrow 1-f),\left(Z_{\mathrm{c}} \rightarrow 1\right)\right]\right|^{2} .
\end{aligned}
$$

This integral can be solved numerically, using the S-wave Coulomb Green's function given in Eq. (A.7) and the regular Coulomb wave function defined in Eq. (9).

The remaining two classes of diagrams to consider at LO evaluate to zero for our choice of reference frame and kinematics. This can be understood from the fact that they correspond to an incoming S-wave. The diagram shown in Fig. 8 evaluates to zero in the zero-momentum frame, since the photon coupling is proportional to the ingoing momentum $\mathbf{p}^{\prime}=0$ of the 
dicluster field:

$$
\begin{aligned}
i \mathcal{A}^{(1)}= & -i \sqrt{\mathcal{Z}} \frac{\nu e\left(Z_{\mathrm{c}}+1\right)}{M_{\mathrm{tot}}} \mathbf{p}^{\prime} i D\left(E, \mathbf{p}^{\prime}\right)(-i g) \\
& \times\left[1+\int \frac{\mathrm{d}^{4} k}{(2 \pi)^{4}} i S_{0}\left(k_{0}, \mathbf{k}\right) i S_{1}\left(E-k_{0},-\mathbf{k}\right) i T_{\mathrm{C}}(\mathbf{k}, \mathbf{p})\right] \\
= & 0
\end{aligned}
$$

The momentum-space amplitude from the next diagrams, which are shown in Fig. 9, is given by

$$
\begin{aligned}
i \mathcal{A}^{(2)}= & -i g \sqrt{\mathcal{Z}} \int \frac{\mathrm{d}^{4} k_{1} \mathrm{~d}^{4} k_{2} \mathrm{~d}^{4} k_{3}}{(2 \pi)^{12}} i S_{0}\left(k_{30}, \mathbf{k}_{3}\right) i S_{1}\left(E-\omega-k_{30},-\mathbf{k}_{3}-\mathbf{Q}\right) \\
& \times i \chi\left(\mathbf{k}_{3}+f \mathbf{Q}, \mathbf{k}_{2}+f \mathbf{Q},-B\right) i S_{0}\left(k_{20}, \mathbf{k}_{2}\right) i S_{1}\left(E-\omega-k_{20},-\mathbf{k}_{2}-\mathbf{Q}\right) \\
& \times\left(-i e Z_{\mathrm{c}}\left(-\mathbf{k}_{2}\right) / m_{1}\right) i S_{1}\left(E-k_{20},-\mathbf{k}_{2}\right) i \chi\left(\mathbf{k}_{2}, \mathbf{k}_{3},-B\right) \\
& \times i S_{0}\left(k_{10}, \mathbf{k}_{1}\right) i S_{1}\left(E-k_{10},-\mathbf{k}_{1}\right)(-i g) i D(E, 0)(-i g) \\
& \times\left[1+\int \frac{\mathrm{d}^{4} k}{(2 \pi)^{4}} i S_{0}\left(k_{0}, \mathbf{k}\right) i S_{1}\left(E-k_{0},-\mathbf{k}\right) i T_{\mathrm{C}}(\mathbf{k}, \mathbf{p})\right] \\
& +\left[(f \rightarrow 1-f),\left(Z_{\mathrm{c}} \rightarrow 1\right),\left(S_{0} \leftrightarrow S_{1}\right)\right] .
\end{aligned}
$$

Doing the same simplifications as before, it can be shown that this amplitude is parallel to the photon momentum:

$$
\begin{aligned}
i \mathcal{A}^{(2)}= & -i g \sqrt{\mathcal{Z}} \int \mathrm{d}^{3} r G_{\mathrm{C}}(-B ; 0, \rho) i \frac{f e Z_{\mathrm{c}}}{m_{\mathrm{R}}} \exp (-i f \mathbf{Q} \cdot \mathbf{r}) \\
& \times\left[\nabla G_{\mathrm{C}}(-B ; \rho, 0)\right](-i g) i D(E, 0)(-i g) \psi_{\mathbf{p}}(0) \\
& +\left[(f \rightarrow 1-f),\left(Z_{\mathrm{c}} \rightarrow 1\right)\right] \\
= & -i g^{3} \sqrt{\mathcal{Z}} D(E, 0) \psi_{\mathbf{p}}(0) \frac{e Z_{\mathrm{c}} f}{m_{\mathrm{R}}} \int \mathrm{d}^{3} r G_{\mathrm{C}}(-B ; 0, \rho) \\
& \times \sum_{l}(2 l+1) i^{l} j_{l}(f \omega r) P_{l}(-\hat{\mathbf{Q}} \cdot \hat{\mathbf{r}})\left[\hat{\mathbf{r}} \frac{\partial}{\partial r} G_{\mathrm{C}}(-B ; \rho, 0)\right] \\
& +\left[(f \rightarrow 1-f),\left(Z_{\mathrm{c}} \rightarrow 1\right)\right] \\
= & \hat{\mathbf{Q}} g^{3} \sqrt{\mathcal{Z}} D(E, 0) \psi_{\mathbf{p}}(0) \frac{e Z_{\mathrm{c}} f}{m_{\mathrm{R}}} \int \mathrm{d}^{3} r G_{\mathrm{C}}(-B ; 0, \rho) j_{1}(f \omega r) \\
& \times\left[\frac{\partial}{\partial r} G_{\mathrm{C}}(-B ; \rho, 0)\right] \\
& +\left[(f \rightarrow 1-f),\left(Z_{\mathrm{c}} \rightarrow 1\right)\right]
\end{aligned}
$$


In the first step the exponential function is expanded in spherical Bessel functions and in the second step all partial waves but $l=1$ integrates to zero. Thus, using Eq. (56),

$$
\epsilon_{i} \cdot \mathcal{A}^{(2)}=0
$$

and the diagram does therefore not contribute.

\subsection{Next-to-leading order}

The higher-order ERE parameters appear with $\nabla+i e \hat{Q} \mathbf{A}$ operators that, in principle, can give contributions to the radiative-capture amplitude. However, the diagrams with these higher-order ERE operators are diagrams with initial-wave scattering due to the strong force. Since we only have included the S-wave part of the strong interaction these initial-wave scattering diagrams are identically zero, which can be understood from the fact that the E1 capture process changes the angular momentum by one. If we were to include also the $\mathrm{P}$-wave interaction explicitly in the field theory, then the effective range, the shape parameter, and so on, would contribute through diagrams with initial P-wave scattering. As the field theory is constructed in this paper, however, the physics of these diagrams is implicitly included in local short-range operators with growing powers of the photon energy $\omega$. Such an operator is explicitly discussed below.

Consequently, there are no additional capture diagrams to consider at NLO. The only contribution at NLO is due to the change in the wavefunction renormalization. This leads to a constant factor

$$
\begin{aligned}
\left(\frac{\sqrt{\mathcal{Z}}}{\sqrt{\mathcal{Z}_{\mathrm{LO}}}}\right)^{2} & =\frac{1}{\tilde{H}\left(\gamma, k_{\mathrm{C}}\right)-3 k_{\mathrm{C}} r_{0}} \\
& =\frac{\tilde{H}\left(\gamma, k_{\mathrm{C}}\right) A^{2}}{6 k_{\mathrm{C}} \Gamma\left(1+k_{\mathrm{C}} / \gamma\right)^{2}}
\end{aligned}
$$

larger (or smaller) result than the LO result. It is important to note that if

$r_{0} \approx \tilde{H}\left(\gamma, k_{\mathrm{C}}\right) /\left(3 k_{\mathrm{C}}\right)$, or equivalently if the ANC $A$ is very large, then the NLO correction will be large, too. We discuss this in more detail in Sec. 6 .

The next correction enters at $\mathrm{N}^{4} \mathrm{LO}$ and as such this calculation is valid up to $\mathrm{N}^{3} \mathrm{LO}$. 


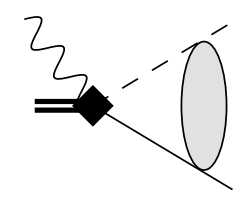

Figure 10: The capture diagram entering at $\mathrm{N}^{4} \mathrm{LO}$. The notation is as in Fig. 1 and time flows from right to left. The diamand vertex corresponds to the short-range interaction of Eq. (71).

\section{3. $N^{4} L O$}

At $\mathrm{N}^{4} \mathrm{LO}$ a short-range E1 operator appears. The interaction term is given by

$\mathcal{L}_{(\mathrm{N} 4 \mathrm{LO})}=\left[D_{5 / 2}^{(\mathrm{E} 1)} \mathcal{C}_{i s}^{a} \mathcal{C}_{a j}^{s^{\prime}}+D_{1 / 2}^{(\mathrm{E} 1)} \mathcal{C}_{i s}^{\sigma} \mathcal{C}_{\sigma j}^{s^{\prime}}\right] d_{s^{\prime}}^{\dagger}\left(\partial_{0} A_{j}-\nabla_{j} A_{0}\right)\left(\psi_{1} \overleftrightarrow{\nabla}_{i} \psi_{0, s}\right)+$ h.c.

and it has dimension 9. This short-range interaction is simply a contact vertex, where the incoming proton-core pair is in a relative $\mathrm{P}$-wave. Note that this operator is not generated by the gauging of any strong interaction terms. However, it is gauge invariant by itself and it connects a $\mathrm{P}$-wave proton-core state with an S-wave dicluster field.

The operator (71) gives rise to the capture diagram in Fig. 10. The tree-level amplitude is given by

$$
\mathcal{B}=D^{(E 1)} \sqrt{\mathcal{Z}} \omega \exp \left(i \sigma_{1}\right) \mathbf{p} \sqrt{\left(1+\eta^{2}\right) C_{\eta}^{2}}
$$

The derivation of the amplitude (72) involves the evaluation of the P-wave integral $\int \frac{\mathrm{d}^{3} k}{(2 \pi)^{3}} \mathbf{k} \psi_{\mathbf{p}}(\mathbf{k})=-\exp \left(i \sigma_{1}\right) \mathbf{p} \sqrt{\left(1+\eta^{2}\right) C_{\eta}^{2}}$, where $\sigma_{1}$ is the pure Coulomb phase shift in the P-wave (see App. B of Ref. [22] for details on P-wave integrals). The symbol $D^{(E 1)}$ has been introduced as a compact notation for the total constant of proportionality.

The next relevant operator

$\mathcal{L}_{(\mathrm{N} 6 \mathrm{LO})}=\left[F_{5 / 2}^{(\mathrm{E} 1)} \mathcal{C}_{i s}^{a} \mathcal{C}_{a j}^{s^{\prime}}+F_{1 / 2}^{(\mathrm{E} 1)} \mathcal{C}_{i s}^{\sigma} \mathcal{C}_{\sigma j}^{s^{\prime}}\right] d_{s^{\prime}}^{\dagger} \partial_{0}\left(\partial_{0} A_{j}-\nabla_{j} A_{0}\right)\left(\psi_{1} \overleftrightarrow{\nabla}_{i} \psi_{0, s}\right)+$ h.c.

of dimension 11 , enters at $\mathrm{N}^{6} \mathrm{LO}$ and as such this calculation is valid up to $\mathrm{N}^{5} \mathrm{LO}$.

It should be noted that if the strong $\mathrm{P}$-wave interaction is included explicitly into the field theory then there would also exist local non-minimal 


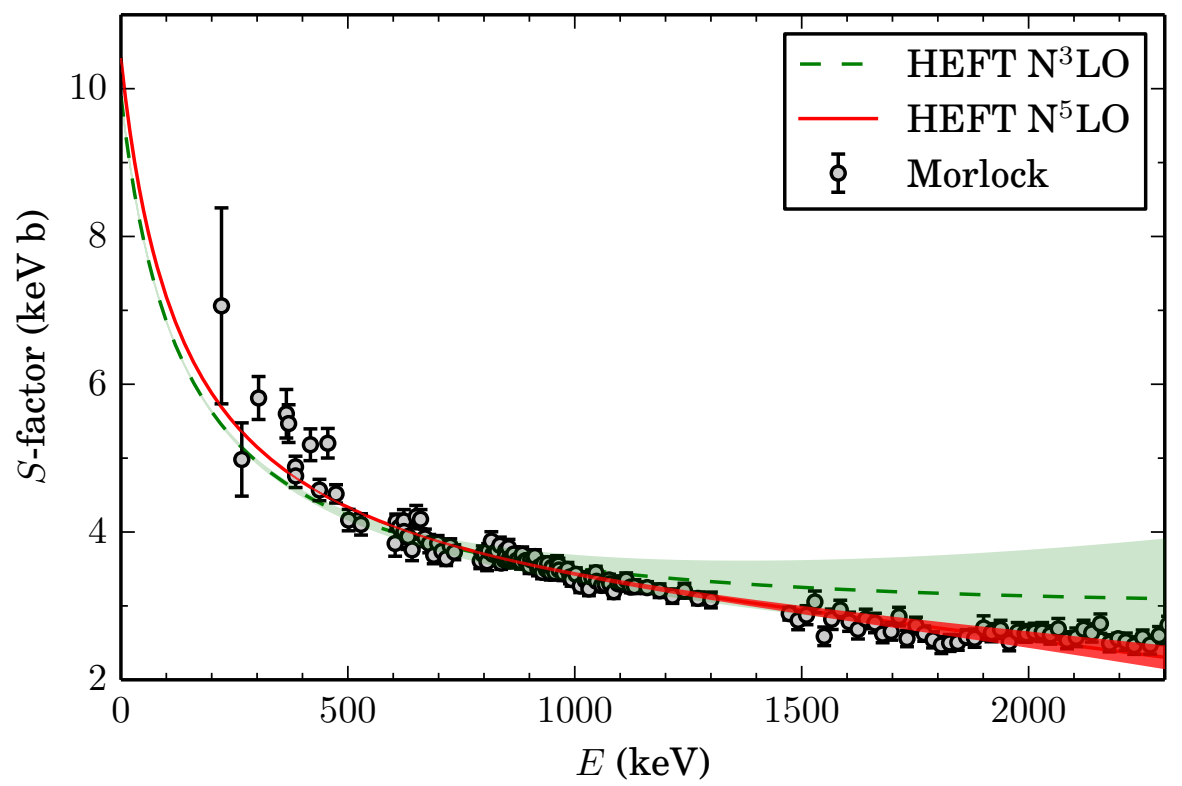

Figure 11: Energy dependent S-factor for ${ }^{16} \mathrm{O}(p, \gamma){ }^{17} \mathrm{~F}^{*}$ fitted to experimental data by Morlock et al. [32]. The error bands correspond to the model error from omitted terms at higher orders. See text for details.

operators of the form [14] $d^{\dagger}\left(\partial_{0} A_{j}-\nabla_{j} A_{0}\right) \tilde{d}$, where $\tilde{d}$ is a P-wave dicluster field (the spin indices on the dicluster fields have been suppressed). If the $\mathrm{P}$-wave is enhanced this operator has scaling dimension 6 and would appear already at NLO. However, as has already been mentioned, we have no reason to suspect that the $\mathrm{P}$-wave interaction is enhanced. As such the calculation can safely be assumed to be accurate up to $\mathrm{N}^{5} \mathrm{LO}$.

\section{S-factor for ${ }^{16} \mathrm{O}(p, \gamma){ }^{17} \mathrm{~F}^{*}$}

The excited $1 / 2^{+}$state of ${ }^{17} \mathrm{~F}$ is due to an S-wave interaction between the valence proton and the ${ }^{16} \mathrm{O}\left(0^{+}\right)$core. As such, we describe this proton halo state using the Halo EFT formalism presented in this paper.

The ANC for this system has been extracted by Huang et al. [33], using a single-particle model fit of radiative capture data, as $A_{1}=77.21 \mathrm{fm}^{-1 / 2}$ and experimentally by Gagliardi et al. [34], using the transfer reaction ${ }^{16} \mathrm{O}\left({ }^{3} \mathrm{He}, d\right){ }^{17} \mathrm{~F}$, as $A_{2}=(80.6 \pm 4.2) \mathrm{fm}^{-1 / 2}$. Comparing these ANCs with the LO result (33), $A_{\mathrm{LO}}=21.4 \mathrm{fm}^{-1 / 2}$, makes it clear that the effective range 
must be very close to the pole position $1 /\left(3 k_{\mathrm{C}}\right)$, such that the LSZ residue becomes large. This implies that the effective range correction for this system is much larger than what one naively expects. This is connected to the fact that $k_{\mathrm{C}} \gg \gamma$ for this system and is discussed in Sec. 6 .

In Fig. 11 we present our Halo EFT results for the radiative proton capture reaction ${ }^{16} \mathrm{O}(p, \gamma){ }^{17} \mathrm{~F}^{*}$ together with data by Morlock et al. [32]. The green dashed line is the Halo EFT result valid up to $\mathrm{N}^{3} \mathrm{LO}$. The single free parameter of this model is the ANC, and this was fitted to the experimental data by Morlock et al. [32] by minimizing an objective function defined as

$$
\chi^{2}(\vec{\alpha}) \equiv \sum_{i \in \mathbb{M}}\left(\frac{\mathcal{O}_{i}^{\text {th }}(\vec{\alpha})-\mathcal{O}_{i}^{\exp }}{\delta_{i}}\right)^{2},
$$

where $\mathcal{O}_{i}^{\text {th }}$ and $\mathcal{O}_{i}^{\text {exp }}$ denote the theoretical and experimental values of observable $\mathcal{O}_{i}$ in the pool of fit data $\mathbb{M}$, and the total uncertainty $\delta_{i}$ determines the weight of the residual. The theoretical values, and therefore the residuals, depend on the vector of fit parameters $\vec{\alpha}$. The total uncertainty is the squared sum of experimental and theoretical errors, $\delta_{i}^{2}=\delta_{\exp , i}^{2}+\delta_{\mathrm{th}, i}^{2}$. The truncation of the Halo EFT expansion allows to estimate the model error. At $\mathrm{N}^{3} \mathrm{LO}$ the omitted terms should scale as $\left(p / k_{\mathrm{hi}}\right)^{4}$, where $p$ is the incoming momentum and $k_{\mathrm{hi}}$ the breakdown scale of the theory. ${ }^{2}$ At this order, we therefore assign a theoretical error $\delta_{\mathrm{th}, i}=C_{\mathrm{EFT}}\left(p_{i} / k_{\mathrm{hi}}\right)^{4}$. Taking these energy-dependent model errors into account will allow us to include relatively high energy data in the fit. We use data up to a center-of-mass energy of $2.3 \mathrm{MeV}$ and determine the amplitude, $C_{\mathrm{EFT}}$, of the model error by the statistical guiding principle that the total $\chi^{2}$ per degree of freedom should be unity. Note that the Morlock data has a normalization error of $10 \%$, which we subtracted in quadrature from the total experimental error during the fitting procedure. This normalization error was added back after the fit had been performed. The constant $C_{\mathrm{EFT}}$ is expected to be of natural size and is determined iteratively such that the $\chi^{2}$ per degree of freedom is minimized to unity. This systematical theory error at $\mathrm{N}^{3} \mathrm{LO}$ is shown as a green band in Fig. 11, with $C_{\mathrm{EFT}}=6.9$ and the breakdown scale given by $k_{\mathrm{hi}}=76 \mathrm{MeV}$. Although somewhat large, this value of $C_{\mathrm{EFT}}$ is still consistent with our power counting estimate.

\footnotetext{
${ }^{2}$ Note that in general the low-momentum scale is given by $\max (p, \gamma)$, but since $p>\gamma$ for all data points in the S-factor fit, here it can simply be taken as $p$.
} 
Similarly, the red line is given by a fit of the $\mathrm{N}^{5} \mathrm{LO}$ model to the same data set where the systematic theory error estimate scales as $\left(p / k_{\mathrm{hi}}\right)^{6}$. At this order we find $C_{\mathrm{EFT}}=1.9$ with $k_{\mathrm{hi}}=76 \mathrm{MeV}$. It is clearly seen that the result converges with increasing order of the EFT and that the S-factor value at threshold is stable. From these fits, we extract a threshold S-factor

$$
S=\left\{\begin{array}{l}
(9.9 \pm 0.1 \text { (stat) } \pm 1.0(\text { norm })) \mathrm{keV} \mathrm{b}, \mathrm{N}^{3} \mathrm{LO} \\
(10.4 \pm 0.1 \text { (stat) } \pm 1.0 \text { (norm) }) \mathrm{keV} \mathrm{b}, \mathrm{N}^{5} \mathrm{LO}
\end{array}\right.
$$

with the $1 \%$ error due to the EFT fit (mainly statistical error) and the $10 \%$ error from the uncertainty in the absolute normalization of the experimental data. These results give the ANC

$$
A=\left\{\begin{array}{l}
(77.4 \pm 0.2(\text { stat }) \pm 3.8(\text { norm })) \mathrm{fm}^{-1 / 2}, \mathrm{~N}^{3} \mathrm{LO} \\
(79.3 \pm 0.2 \text { (stat) } \pm 3.9(\text { norm })) \mathrm{fm}^{-1 / 2}, \mathrm{~N}^{5} \mathrm{LO}
\end{array}\right.
$$

which is consistent with the ANCs of Huang et al. and Gagliardi et al..

The charge radius of the ${ }^{17} \mathrm{~F}^{*}$ can now be obtained by using an extracted ANC. Using the ${ }^{16} \mathrm{O}$-proton ANC extracted from the $\mathrm{N}^{5} \mathrm{LO}$ radiative proton capture fit, the resulting NLO charge radius is given by

$$
r_{\mathrm{C}, \mathrm{NLO}}=(2.20 \pm 0.04(\mathrm{EFT}) \pm 0.11(\mathrm{ANC})) \mathrm{fm} .
$$

The NLO EFT error in Eq. (77) was estimated from the EFT expansion parameter squared, $\left(\gamma / k_{\mathrm{hi}}\right)^{2}$, using a breakdown scale $k_{\mathrm{hi}}=76 \mathrm{MeV}$, which is of the same order as the inverse core radius $1 / R_{\text {core }} \sim 73 \mathrm{MeV}$. The dominant error in Eq. (77) is from the normalization error of the Morlock data, through the extracted ANC. However, there could also be additional EFT errors for this result due to the non-inclusion of the operators that are responsible for the finite-size contributions of the constituents [35]. Alternatively, we can interpret our result as the radius relative to the ${ }^{16} \mathrm{O}$ core.

\section{Fine tuning and S-wave proton halos}

Along the neutron drip-line there exist several neutron halo states. These states are characterized by an unnaturally large neutron-core scattering length, which brings the state very close to threshold. However, proton halos are much more rare. In the S-wave case this can be understood by considering the Coulomb repulsion between the valence proton and the core. For a proton 


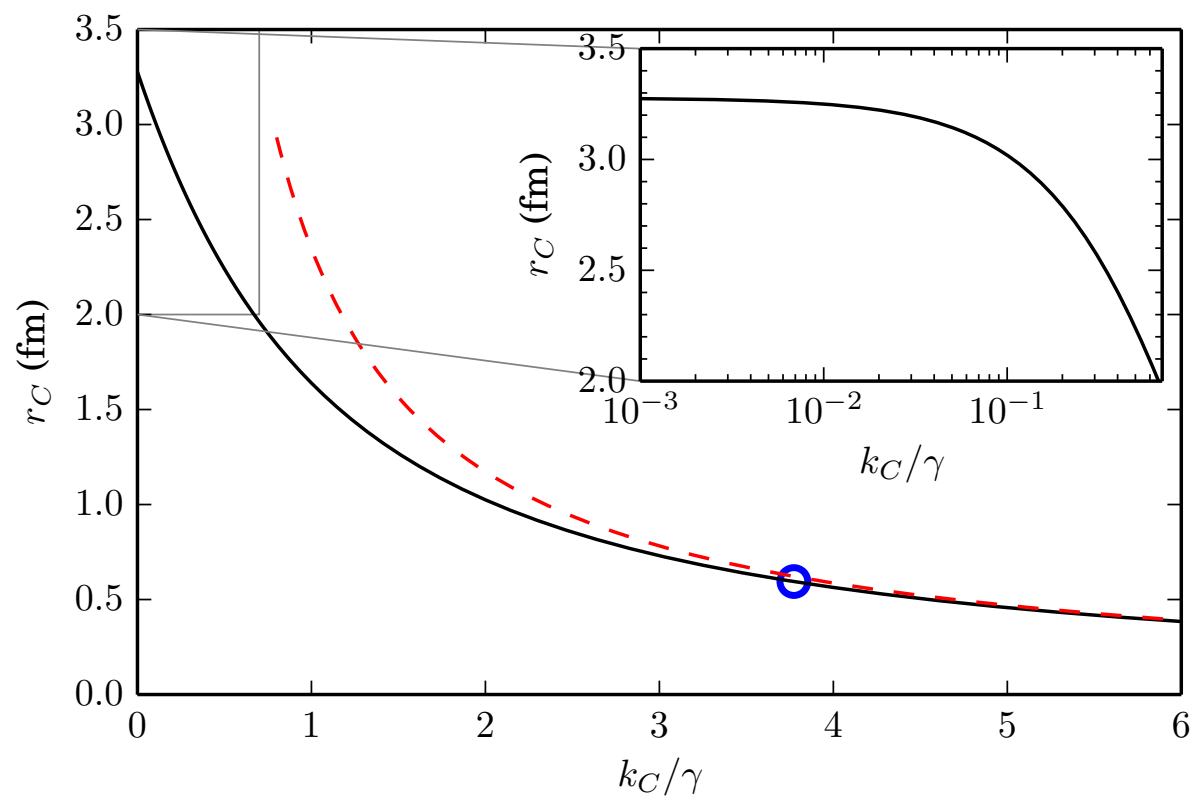

Figure 12: The dependence of the LO charge radius on $k_{\mathrm{C}} / \gamma$. The solid black line is the LO Halo EFT result, the blue circle denotes the ${ }^{17} \mathrm{~F}^{*}$ system, and the dashed red line is the asymptotic $1 / k_{\mathrm{C}}$ behavior. The inset shows the low-energy region in a semi-logarithmic scale, illustrating the hypothetical neutron halo limit (78). The curve was generated using a binding momentum $\gamma=13.6 \mathrm{MeV}$.

halo state to exist, we need the attractive strong force to be almost cancelled by the Coulomb repulsion, resulting in a threshold state [30, 31]. This cancellation can be seen within our formalism as an additional fine-tuning in the effective range. Note that for an S-wave proton halo state this means that the proton-core scattering length needs to be unnaturally large and that the effective range must be fine-tuned to cancel the Coulomb repulsion. The existence of proton halos is therefore doubly suppressed by the need for two fine-tunings.

Proton halo systems contain the second scale $k_{\mathrm{C}}$ that will depend on the charge of the degrees of freedom in the system. Within our framework, $k_{\mathrm{C}}$ is a parameter that is independent of the Coulomb-modified effective range parameters. When $k_{\mathrm{C}} / \gamma \gg 1$, we will speak of the extreme Coulomb regime. In this regime the two particles tend to be close together, since otherwise the system is ripped apart by the Coulomb repulsion. This limit is in practice 
realized for the ${ }^{17} \mathrm{~F}^{*}$ system, where $k_{\mathrm{C}} / \gamma=3.8$. In Fig. 12 the LO charge radius is shown as a function of the Sommerfeld parameter $k_{\mathrm{C}} / \gamma$, where we have used the binding momentum for ${ }^{17} \mathrm{~F}^{*}, \gamma=13.6 \mathrm{MeV}$. The blue circle is the parameter point corresponding to the physical ${ }^{17} \mathrm{~F}^{*}$ system. It is clear that this system is almost in the extreme Coulomb regime. Note that the resulting LO charge radius is very small for a strong Coulomb repulsion, since it has an asymptotic $1 / k_{\mathrm{C}}$ behavior. At the far left, where $k_{\mathrm{C}} \ll \gamma$, the system mimics that of a neutron halo, with the only difference being that the photon also can couple through minimal substitution to the nucleon field. The limiting value for $k_{\mathrm{C}} / \gamma \rightarrow 0$ is therefore given by [14]

$$
\lim _{k_{\mathrm{C}} / \gamma \rightarrow 0} r_{\mathrm{C}}^{2}=\frac{1}{Z_{\mathrm{c}}+1} \frac{Z_{\mathrm{c}} f^{2}+(1-f)^{2}}{2 \gamma^{2}} .
$$

In the standard power counting for systems with large S-wave scattering length the effective range enters at NLO. The hierarchy of scales in this case is $\gamma, k_{\mathrm{C}} \ll 1 / r_{0} \sim 1 / R_{\text {core }}$, where $R_{\text {core }}$ is the length scale set by the core. However, the discussion above implies that we can have $k_{\mathrm{C}} r_{0} \sim 1$ instead of $k_{\mathrm{C}} \ll 1 / r_{0}$. In the zero-range limit, the inverse Coulomb momentum sets the scale for the LO charge radius and the effective range contributions will therefore be numerically large since the LSZ-factor for S-wave proton halo nuclei with the effective range included behaves as

$$
\mathcal{Z} \propto \frac{1}{1-3 k_{\mathrm{C}} r_{0}} .
$$

It appears to be fine tuned to the pole position with $r_{0} \sim 1 /\left(3 k_{\mathrm{C}}\right)$. In the case of the ${ }^{17} \mathrm{~F}^{*}$ system the effective-range correction results in a factor 3.63.8 larger charge radius. This hierarchy-of-scales problem can be solved by fixing the bound state pole position of the t-matrix at leading order and the ANC at NLO. This procedure ensures that corrections beyond NLO scale naturally again and is similar in spirit to the so-called Z-parameterization as introduced in Ref. [37].

\section{Conclusions}

We have calculated the charge radius and radiative capture cross section for proton halo nuclei interacting through a large S-wave scattering length. Specifically, we have included higher-order effective-range corrections and 
shown consequent good agreement with experimental data. Our description of proton-capture on ${ }^{16} \mathrm{O}$ agrees very well with the data and leads to a new way of extracting the low-energy S-factor and the corresponding ANC with error estimates that take the intrinsic effective theory error into account. For the excited state of ${ }^{17} \mathrm{~F}$ we found a large correction to all observables at NLO and have explained this as a result of the complicated interplay between the range of the strong interaction and the scale set by the Coulomb interaction. We showed that constraining LO and NLO to proton bound state pole position and ANC will warrant that higher order corrections scale naturally. Our results highlight that Halo EFT provides a powerful tool to analyze experimental data and predict observables with theoretical error estimates. A further advantage of the analysis of such systems with an EFT framework is the proper identification of two-body contributions to the electromagnetic current operator. The physics of such terms should in principle also appear in a cluster model description although there are some contributions that are not generated by gauging the strong interaction terms and are gauge invariant by themselves. The EFT power counting also provides a reliable estimate for the relative importance of these terms.

Our analysis can also be used to obtain the low-energy phaseshifts for nucleon-nucleus elastic scattering that can then be compared to corresponding ab initio calculations that have become possible over the last years [36]. For this, however, explicit higher-oder calculations relating the exact ANC to the effective range parameters for nucleon-nucleus scattering would be required.

It is a very interesting question, whether a two-proton halo could in principle support an effective three-body bound state and how the spectrum of this system compares to two-neutron halo nuclei bound by resonant Swave interactions. This question is also relevant for studies of two-proton radioactivity $[38,39]$. Furthermore, it seems worthwhile to investigate the description of low-lying resonances in proton+core systems.

\section{Acknowledgment}

We thank H. Esbensen and S. König for helpful discussions, and P. Mohr for supplying relevant data. This work was supported by the Swedish Research Council (dnr. 2010-4078), the European Research Council under the European Communitys Seventh Framework Programme (FP7/2007-2013) / ERC grant agreement no. 240603, the Swedish Foundation for International 
Cooperation in Research and Higher Education (STINT, Grant No. IG20125158), the Office of Nuclear Physics, U.S. Department of Energy under Contract nos. DE-AC02-06CH11357 and DE-AC05-00OR22725, by the BMBF under contracts 05P12PDFTE and 05P15RDFN1, and by the Helmholtz Association under contract HA216/EMMI.

\section{Appendix A. Partial wave projected Coulomb Green's function}

In this appendix, we discuss the construction and partial wave projection in the S-wave channel.

It is convenient to use the Coulomb Green's function in a non-integral form and below we present such a form for the bound state Green's function. This can be done by doing a partial-wave projection and forming the Green's function as a product between two independent Coulomb wavefunctions, satisfying one boundary condition each, in accordance with the definition of Green's function. For the $r=0$ boundary condition we must use the regular Coulomb wave function $F_{L}$ and to satisfy the condition for a bound state at $r=\infty$ we need to form the combination

$$
i F_{L}+G_{L}
$$

This can be seen from the asymptotics

$$
F_{L}(\eta, \rho) \rightarrow \sin \left(\rho-L \pi / 2-\eta \log (2 \rho)+\sigma_{L}\right)
$$

and

$$
G_{L}(\eta, \rho) \rightarrow \cos \left(\rho-L \pi / 2-\eta \log (2 \rho)+\sigma_{L}\right),
$$

using that for a bound state $\rho=i \gamma r$, with $\gamma>0$, where the only combination that yields only an $\exp (-\gamma r)$ dependence is the combination given in (A.1). Therefore, the partial wave projected Coulomb Green's function is

$$
G_{\mathrm{C}}^{(L)}\left(-B ; \rho^{\prime}, \rho\right)=-\frac{m_{\mathrm{R}} p}{2 \pi} \frac{F_{L}\left(\eta, \rho^{\prime}\right)\left[i F_{L}(\eta, \rho)+G_{L}(\eta, \rho)\right]}{\rho^{\prime} \rho},
$$

where the normalization is given by the Wronskian of the Coulomb wavefunctions and the Coulomb-Schrödinger equation.

For the S-wave bound state observables we consider, only propagation with a Coulomb Green's function down to zero separation is needed, that is 
$G_{\mathrm{C}}^{(0)}(-B ; 0, r)$. Using that

$i F_{L}(\eta, \rho)+G_{L}(\eta, \rho)=\sqrt{\frac{\Gamma(L+1+i \eta)}{\Gamma(L+1-i \eta)}} \exp \left(-i \frac{\pi}{2}(L+i \eta)\right) W_{-i \eta, L+1 / 2}(-2 i \rho)$,

which was shown in [27], and the limit

$$
\lim _{\rho \rightarrow 0} \frac{F_{0}(\eta, \rho)}{\rho}=\exp (-\pi \eta / 2) \sqrt{\Gamma(1+i \eta) \Gamma(1-i \eta)}
$$

we may write Eq. (A.4) for S-waves as

$$
G_{\mathrm{C}}^{(0)}(-B ; 0, \rho)=-\frac{m_{\mathrm{R}} p}{2 \pi} \Gamma(1+i \eta) \frac{W_{-i \eta, 1 / 2}(-2 i \rho)}{\rho}
$$

\section{References}

[1] M. V. Zhukov, B. V. Danilin, D. V. Fedorov, J. M. Bang, I. J. Thompson and J. S. Vaagen, Phys. Rept. 231, 151 (1993).

[2] K. Riisager, Rev. Mod. Phys. 66 (1994) 1105.

[3] A. S. Jensen, K. Riisager, D. V. Fedorov and E. Garrido, Rev. Mod. Phys. 76 (2004) 215.

[4] P. Hansen, A. Jensen and B. Jonson, Ann. Rev. Nucl. Part. Sci. 45 (1995) 591.

[5] C. A. Bertulani, H.-W. Hammer and U. Van Kolck, Nucl. Phys. A 712, 37 (2002).

[6] P. F. Bedaque, H.-W. Hammer and U. van Kolck, Phys. Lett. B 569 (2003) 159.

[7] J. Rotureau and U. van Kolck, Few Body Syst. 54 (2013) 725.

[8] C. Ji, C. .Elster and D. R. Phillips, Phys. Rev. C 90 (2014) 044004.

[9] D. L. Canham and H. -W. Hammer, Eur. Phys. J. A 37 (2008) 367.

[10] G. Rupak and R. Higa, Phys. Rev. Lett. 106 (2011) 222501. 
[11] L. Fernando, R. Higa and G. Rupak, Eur. Phys. J. A 48 (2012) 24.

[12] X. Zhang, K. M. Nollett and D. R. Phillips, Phys. Rev. C 89 (2014) 024613.

[13] P. Hagen, H. -W. Hammer and L. Platter, Eur. Phys. J. A 49 (2013) 118.

[14] H. -W. Hammer and D. R. Phillips, Nucl. Phys. A 865 (2011) 17.

[15] G. Rupak, L. Fernando and A. Vaghani, Phys. Rev. C 86 (2012) 044608.

[16] B. Acharya and D. R. Phillips, Nucl. Phys. A 913 (2013) 103.

[17] B. Acharya, C. Ji and D. R. Phillips, Phys. Lett. B 723 (2013) 196.

[18] G. Hagen, P. Hagen, H. -W. Hammer and L. Platter, Phys. Rev. Lett. 111 (2013) 13, 132501.

[19] X. Kong and F. Ravndal, Phys. Lett. B 450 (1999) 320.

[20] E. Ryberg, C. Forssén, H. W. Hammer and L. Platter, Phys. Rev. C 89 (2014) 014325.

[21] X. Zhang, K. M. Nollett and D. Phillips, Phys. Rev. C 89 (2014) 051602.

[22] E. Ryberg, C. Forssén, H. W. Hammer and L. Platter, Eur. Phys. J. A 50 (2014) 170.

[23] R. Higa, H.-W. Hammer and U. van Kolck, Nucl. Phys. A 809 (2008) 171.

[24] U. van Kolck, Nucl.Phys. A 645 (1999) 273.

[25] D. B. Kaplan, M. J. Savage and M. B. Wise, Phys. Lett. B 424 (1998) 390.

[26] D. B. Kaplan, M. J. Savage and M. B. Wise, Nucl. Phys. B 534 (1998) 329.

[27] S. Koenig, D. Lee and H.-W. Hammer, J. Phys. G: Nucl. Part. Phys. 40 (2013) 045106. 
[28] H. A. Bethe, Phys. Rev. 76 (1949) 38.

[29] J. C. Y. Chen and A. C. Chen, Adv. At. Mol. Phys. 8 (1972) 71.

[30] M. Zhukov and I. Thompson, Phys.Rev. C 52 (1995) 3505.

[31] P. Woods and C. Davids, Ann. Rev. Nucl. Part. Sci. 47 (1997) 541.

[32] R. Morlock, R. Kunz, A. Mayer, M. Jaeger, A. Müller, J. Hammer, P. Mohr, H. Oberhummer, G. Staudt and V. Kölle, Phys. Rev. Lett. 79 (1997) 3837.

[33] J. Huang, C. Bertulani and V. Guimaraes, Atom. Data Nucl. Data Tabl. 96 (2010) 824.

[34] C. Gagliardi, R. Tribble, A. Azhari, H. Clark, Y. Lui, et al., Phys. Rev. C 59 (1999) 1149.

[35] E. Ryberg, C. Forssén and U. van Kolck, in preparation.

[36] G. Hagen and N. Michel, Phys. Rev. C 86 (2012) 021602.

[37] D. R. Phillips, G. Rupak and M. J. Savage, Phys. Lett. B 473, 209 (2000).

[38] L. Grigorenko, R. Johnson, I. Mukha, I. Thompson and M. Zhukov, Phys. Rev. Lett. 85 (2000) 22.

[39] B. Blank and M. Ploszajczak, Rept. Prog. Phys. 71 (2008) 046301. 